\title{
CORRIGENDUM TO ON THE SIMPLICITY OF SOME CUNTZ-PIMSNER ALGEBRAS
}

\author{
PAUL S. MUHLY and BARUCH SOLEL
}

We are grateful to Dr. Jürgen Schweizer for pointing out to us that Theorem 1 in [1] is incorrect. It rests upon a faulty application in Lemma 8 of hypothesis (H5). Hypothesis (H5) asserts that $\Omega\left(1_{A}\right) \neq 1_{A}$ and we concluded at the end of the proof of Lemma 8 that $\tau_{0}\left(\Omega\left(1_{A}\right)\right) \neq 1$ for a particular faithful tracial state $\tau_{0}$. This inequality is a critical point of the proof. However, the following example, due to Dr. Schweizer, shows that this conclusion is faulty. Let $E$ be the identity correspondence over $A=M_{2}(\mathrm{C})$, and let

$$
\eta_{1}=\left(\begin{array}{ll}
0 & 0 \\
0 & 1
\end{array}\right) \quad \text { and } \quad \eta_{2}=\left(\begin{array}{ll}
0 & 1 \\
0 & 0
\end{array}\right)
$$

The map

$$
a \rightarrow\left(\begin{array}{l}
\left\langle a, \eta_{1}\right\rangle_{A} \\
\left\langle a, \eta_{2}\right\rangle_{A}
\end{array}\right)
$$

embeds $E=A=M_{2}(\mathrm{C})$ into $C^{2}\left(M_{2}(\mathrm{C})\right)$ as a direct summand, and

$$
\Omega(a)=\left\langle\eta_{1}, a \eta_{1}\right\rangle+\left\langle\eta_{2}, a \eta_{2}\right\rangle=\left(\begin{array}{cc}
0 & 0 \\
0 & a_{11}+a_{22}
\end{array}\right), \quad a=\left(\begin{array}{ll}
a_{11} & a_{12} \\
a_{21} & a_{22}
\end{array}\right) .
$$

Therefore,

$$
\Omega\left(1_{A}\right)=\left(\begin{array}{ll}
0 & 0 \\
0 & 2
\end{array}\right)
$$

On the other hand, $M_{2}(\mathrm{C})$ has only one tracial state, $\tau_{0}$, the normalized trace, and $\tau_{0}\left(\Omega\left(1_{A}\right)\right)=1$. Note, too, that all the other hypotheses of Theorem 1 in [1] are satisfied, but that $\mathscr{O}(E) \simeq M_{2}(\mathrm{C}) \rtimes_{\text {id }} \mathrm{Z} \simeq M_{2}(\mathrm{C}) \otimes C\left(S^{1}\right)$ is not simple. 
We offer the following stronger hypothesis that bridges the gap in the proof of [1, Theorem 1] and which is still satisfied in all of the examples where Theorem 1 is applied.

Hypothesis $(\mathrm{H} 5 *)$. Among all tracial states $\tau$ on $A$ that are scaled by $\Omega$, i.e., that satisfy the equation $\tau \circ \Omega=c \tau$, for some $c \in \mathbf{R}$, there is at least one with $c \neq 0,1$.

Of course, if $\Omega$ scales $\tau$, then $c=\tau\left(\Omega\left(1_{A}\right)\right)$. Also, evidently, hypothesis $(\mathrm{H} 5 *)$ implies hypothesis (H5). Note that Lemma 6 of [1], which uses only hypotheses (H1)-(H4), shows that the set of faithful traces that are scaled by $\Omega$ is non-empty. Further, under hypothesis (H4), any trace that is scaled by $\Omega$ must be faithful. Thus, under hypothesis (H4), one can never have $c=$ $\tau\left(\Omega\left(1_{A}\right)\right)=0$.

It is hypothesis $\left(\mathrm{H} 5^{*}\right)$ that is used on page 63 at the end of the proof Lemma 8 and this is the only place hypothesis (H5*) is used anywhere in the proof of Theorem 1. Thus, the details that we present in [1] prove

THeOREM 0.1. If the $C^{*}$-algebra $A$ and the correspondence $E$ satisfy hypotheses $(H 1)-(H 4)$ of [1] and hypothesis $\left(H 5^{*}\right)$, then $\mathscr{O}(E)$ is simple.

Observe that if $\Omega$ satisfies the condition that $\Omega\left(1_{A}\right)$ is comparable with $1_{A}$, but not equal to $1_{A}$, then necessarily hypothesis (H5*) is satisfied under hypotheses (H1)-(H4). The reason, as we just noted, is that Lemma 6 implies that every trace that is scaled by $\Omega$ necessarily is faithful, under hypotheses (H1)-(H4), and every faithful trace satisfies the equation $\tau\left(\Omega\left(1_{A}\right)\right) \neq 1$ if $\Omega\left(1_{A}\right)$ is comparable with, but different from $1_{A}$. In Corollary 2, the map $\Omega$ satisfies the condition $\Omega\left(1_{A}\right) \lesseqgtr 1_{A}$, while in Corollary $14, \Omega\left(1_{A}\right)=n 1_{A}$, $n \geq 2$. Thus, these two corollaries remain valid with no change in hypotheses. It remains to prove Corollary 3 .

There is no problem with the verifications in [1] that hypotheses (H1)-(H4) are satisfied. We show that there is a faithful trace $\tau_{0}$ on $A$ that is scaled by $\Omega$ with $\tau_{0}\left(\Omega\left(1_{A}\right)\right) \neq 1$. To this end, let $M$ be the set of all tracial states on $B$ that are $\Phi$-invariant. One of the hypotheses of Corollary 3 is that $M$ is non-empty. We define the completely positive map $\beta$ on $B$ by the formula $\beta(b)=\sum \Phi\left(u_{i}^{*} b u_{i}\right)$ where $\left\{u_{i}\right\}$ is the quasi-basis, $u_{i}=W^{-1} P \varepsilon_{i}$ used in [1]. (Recall that for $x \in B, W x=\left(\Phi\left(u_{1}^{*} x\right), \Phi\left(u_{2}^{*} x\right), \ldots, \Phi\left(u_{n}^{*} x\right)\right)^{t}$.) Observe that for $b \in A, \beta(b)=\sum\left\langle u_{i}, b u_{i}\right\rangle=\sum\left\langle P \varepsilon_{i}, W \varphi(b) W^{-1} P \varepsilon_{i}\right\rangle=\Omega(b)$. For $\rho \in M$, write

$$
\rho^{\prime}(b)=\left(\rho\left(\beta\left(1_{B}\right)\right)\right)^{-1} \rho(\beta(b)),
$$

$b \in B$. (Observe that if we apply the argument in the first paragraph of the proof of [1, Lemma 6] to the restriction $\rho \mid A$ and note that $1_{B}=1_{A}$, we find 
that $\rho\left(\beta\left(1_{B}\right)\right)=\rho\left(\beta\left(1_{A}\right)\right)=\rho\left(\Omega\left(1_{A}\right)\right) \neq 0$. Thus $\rho^{\prime}$ is well defined.) Then $\rho^{\prime}$ is a $\Phi$-invariant state on $B$. To show that $\rho^{\prime}$ is a trace, let $b, c \in B$. Then because $\rho$ is a $\Phi$-invariant trace, we have $\rho(\beta(b c))=\sum \rho\left(\Phi\left(u_{i}^{*} b c u_{i}\right)\right)=$ $\rho\left(\sum u_{i}^{*} b c u_{i}\right)=\rho\left(\sum u_{i} u_{i}^{*} b c\right)=\rho(($ ind $\Phi) b c)$, where ind $\Phi=\sum u_{i} u_{i}^{*}$. Likewise, we see that $\rho(\beta(c b))=\rho(($ ind $\Phi) c b)$. Since $\rho$ is a trace and (ind $\Phi)$ lies in the center of $B$, we see that $\rho \circ \beta$ is tracial. Therefore $\rho^{\prime}$ is a trace; i.e., $\rho^{\prime} \in M$. By the Schauder fixed point theorem, there is a $\rho_{0} \in M$ such that $\rho_{0}=\rho_{0}^{\prime}$, i.e., $\rho_{0}(b) \rho_{0}\left(\beta\left(1_{B}\right)\right)=\rho_{0}(\beta(b))$. If we set $\tau_{0}=\rho_{0} \mid A$, then since $\Omega=\beta \mid A$, we see that $\Omega$ scales $\tau_{0}$. To verify that hypothesis (H5*) is satisfied, we need to show $\tau_{0}\left(\Omega\left(1_{A}\right)\right) \neq 0,1$. To show $\tau_{0}\left(\Omega\left(1_{A}\right)\right) \neq 0$, it suffices to show that $\rho_{0}$ is faithful. Observe that since $\rho_{0}$ is $\Phi$-invariant, we may write $\rho_{0}=\tau_{0} \circ \Phi$. Set $N_{\rho_{0}}=\left\{b \in B \mid \rho_{0}\left(b^{*} b\right)=0\right\}$. Then $N_{\rho_{0}}$ is a two-sided ideal in $B$ which $\Phi$ maps into the two-sided ideal $N_{\tau_{0}}:=\{a \in A \mid$ $\left.\tau_{0}\left(a^{*} a\right)=0\right\}$ in $A$. (Indeed, for $b \in N_{\rho_{0}}, \tau_{0}\left(\Phi(b)^{*} \Phi(b)\right) \leq \tau_{0}\left(\Phi\left(b^{*} b\right)\right)=$ $\rho_{0}\left(b^{*} b\right)=0$, by the Cauchy-Schwarz-Kadison inequality.) However, $N_{\tau_{0}}$ is $\Omega$-invariant, since $\Omega$ scales $\tau_{0}$ (see the proof of [1, Lemma 6]). By hypothesis (H4), $N_{\tau_{0}}=\{0\}$, and since $\Phi$ is faithful by [4, Proposition 2.1.5], we see that $N_{\rho_{0}}=\{0\}$, too. Thus $\rho_{0}$ is faithful. To see that $\tau_{0}\left(\Omega\left(1_{A}\right)\right) \neq 1$, observe that $\tau_{0}\left(\Omega\left(1_{A}\right)\right)=\sum \tau_{0}\left(\Phi\left(u_{i}^{*} u_{i}\right)\right)=\sum \rho_{0}\left(u_{i}^{*} u_{i}\right)=\sum \rho_{0}\left(u_{i} u_{i}^{*}\right)=\rho_{0}($ ind $\Phi)$. By [4, Lemma 2.3.1], ind $\Phi$ is an element of the center of $B$ that dominates 1. Further, since $A \neq B$, by hypothesis, ind $\Phi \neq 1$ by [4, Proposition 2.3.7]. Therefore, $\tau_{0}\left(\Omega\left(1_{A}\right)\right)=\rho_{0}$ (ind $\left.\Phi\right) \gtreqless 1$.

Finally, we note that Theorem 5 of [1], and the results related to it, are unaffected by the change from hypothesis (H5) to hypothesis (H5*).

For what appears to be the final word on the simplicity issue for $\mathscr{O}(E)$, please consult [2], [3].

\section{REFERENCES}

1. Muhly, P. and Solel, B., On the simplicity of some Cuntz-Pimsner algebras, Math. Scand. 83 (1998), 53-73.

2. Schweizer, J., Crossed Products by $C^{*}$-correspondences and Cuntz-Pimsner algebras, $C^{*}$ algebras (Münster, 1999), 203-226, Springer, Berlin, 2000.

3. Schweizer, J., Dilations of $C^{*}$-correspondences and the simplicity of Cuntz-Pimsner algebras, J. Funct. Anal. 180 (2001), 404-425.

4. Watatani, Y., Index for $C^{*}$-subalgebras, Mem. Amer. Math. Soc. 83 (\#424), 1990.

DEPARTMENT OF MATHEMATICS

UNIVERSITY OF IOWA

IOWA CITY, IOWA 52242-1419

USA
DEPARTMENT OF MATHEMATICS

TECHNION

32000 HAIFA

ISRAEL 